\title{
Frequency of human Parvovirus B19 among patients with respiratory infection in Iran
}

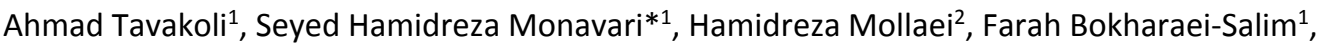 \\ Maryam Esghaei ${ }^{1}$, Hossein Keyvani ${ }^{1}$, Hadi Ghaffari ${ }^{1}$
}

\begin{abstract}
Background: Human parvovirus B19 was known as one of the possible cause of mild respiratory tract diseases in previous studies. However, there are some reports of acute obstructive respiratory disease and severe pneumonia. The purpose of current study was to assess the prevalence and clinical features of parvovirus B19 in respiratory infection.

Methods: This study was conducted on 156 patients diagnosed with respiratory infection at the Iran University of Medical Sciencesaffiliated hospitals. After extraction of viral DNA from swab samples, detection of parvovirus B19 was performed by real-time PCR assay.

Results: In 156 patient's samples, parvovirus B19 was found in 8 (5.1\%) cases including 5 males (5.9\%) and 3 females (4.1\%). The most common clinical symptoms were wheezing (100\%), tachypnea (100\%), fever (75\%) and rhinorrhea/pharyngitis $(75 \%)$.

Conclusion: This is the first attempt to assess the prevalence of parvovirus B19 infection in Iranian patients with respiratory infection. The low frequency of parvovirus B19 detected in our study does not support the role of this virus in the development of respiratory infection. However, further studies are needed to better evaluate the etiological role of parvovirus B19 in respiratory infection.
\end{abstract}

Keywords: Parvovirus B19, Real-time PCR, Respiratory infection, Iran

Copyright $₫$ Iran University of Medical Sciences

Cite this article as: Tavakoli A, Monavari SH, Mollaei H, Bokharaei-Salim F, Esghaei M, Keyvani H, Ghaffari H. Frequency of human Parvovirus B19 among patients with respiratory infection in Iran. Med J Islam Repub Iran. 2018(8 May);32:38. https://doi.org/10.14196/mjiri.32.38

\section{Introduction}

Human parvovirus B19 is an extensively studied member of the genus erythroparvovirus of the family Parvoviridae. Patients infected by the human parvovirus B19 can be asymptomatic or symptomatic. In some cases, the virus is associated with serious clinical conditions such as erythema infectiosum, arthropathy, hydrops fetalis, transient aplastic crisis (TAC), idiopathic thrombocytopenic purpura, and chronic red cell aplasia. Humans are the only known host for parvovirus B19 (1-3).

Identification of B19 relies on IgM and IgG antibodies detection using ELISA test and also, detection of viral

Corresponding author: Dr Seyed Hamidreza Monavari, hrmonavari@yahoo.com

1. Department of Virology, Faculty of Medicine, Iran University of Medical Sciences, Tehran, Iran.

2. Department of Microbiology and Virology, Faculty of Medicine, Kerman University of Medical Sciences, Kerman, Iran.
DNA by PCR assay (4). Although IgM antibody against parvovirus B19 is normally detectable only for a few months, IgG parvovirus B19 antibody persists for many years or lifelong (5). Erythema infectiosum is generally self-limited and requires no specific therapy. However, in patients with transient aplastic crises, immunodeficiency and anemia, transfused red blood cells and intravenous immunoglobulin have been proposed, respectively (6).

Previous studies in volunteers demonstrated that parvovirus B19 first replicates in the nasopharynx or the upper respiratory tract and then spreads by viremia to the bone

$\uparrow$ What is "already known" in this topic:

A few studies have examined the association between pneumonia, self-limited obstructive airway disease, or pleural effusions and human parvovirus B19 infection. This hypothesis has evolved from the fact that in addition to erythroid cells, the cellular receptor for parvovirus B19 has been shown to be expressed in other tissues such as the lung. However, so far no studies have linked parvovirus B19 to respiratory tract diseases.

$\rightarrow$ What this article adds:

Our findings show that low-frequency (5.1\%) parvovirus B19 genome have detected in throat swab specimens taken from patients with different symptoms of respiratory infection. However, further extensive investigations are essential to support the etiological role of parvovirus B19 in respiratory infection. 
marrow and elsewhere, where it replicates and destroys erythroid precursor cells (7). Human parvovirus B19 can cause a mild respiratory tract illness with no rash. There are some reports of acute obstructive respiratory disease, severe pneumonia, self-limited obstructive airway disease, and pleural effusions in patients with B19 infection (5, 810). However, studies reporting the prevalence of B19 infection in patients suffering from respiratory infection are scanty. Hence, the aim of the current study was to evaluate the frequency of B19 infection in this population.

\section{Methods}

\section{Patients and Specimen Collection}

From January to June 2014, throat swab samples were collected from 156 patients diagnosed with respiratory infections at hospitals affiliated to the Iran University of Medical Sciences, Tehran, Iran. Informed consent was obtained from all patients included in the study. The diagnosis of respiratory disease made by physicians, defined with symptoms of pneumonia, bronchiolitis, asthma and acute nasopharyngitis. The exclusion criteria were patients with immune deficiency conditions such as chemotherapy, immunosuppressive therapy or bone marrow transplantation. Samples were collected from each patient and stored in a viral transport medium and after transporting to the Virology Laboratory of Iran University of Medical Sciences, immediately stored at $-70^{\circ} \mathrm{C}$. All patients' medical records, demographic and clinical data including detailed signs and symptoms and laboratory results were collected. This study followed the principles of the Declaration of Helsinki and was approved by the local ethics committee of the Iran University of Medical Sciences, Tehran, Iran (Ethics Number: 25415).

\section{Nucleic acid Extraction}

Viral nucleic acid extraction was performed using Invisorb spin virus DNA Mini Kit (Invitek, Berlin, Germany) according to the manufacturer's instructions, and extracted
DNA was stored at $-20^{\circ} \mathrm{C}$ until use.

\section{Real-time Polymerase Chain Reaction}

The real-time PCR assay was performed using the RotorGene Q instrument (Qiagen, Germany) for the detection of parvovirus B19. The primers and TaqMan probe were obtained from our previous study (1) which designed in the NS1 region, with the most conserved region among different strains of parvovirus B19. The amplification conditions were as follows: $95^{\circ} \mathrm{C}$ for $10 \mathrm{~min}$, then 40 cycles of the following sequential steps: $95^{\circ} \mathrm{C}$ for $15 \mathrm{~s}, 60^{\circ} \mathrm{C}$ for $1 \mathrm{~min}$.

\section{Statistical Analyses}

All statistical analysis was performed using SPSS v.21.0. Categorical variables were compared by Fisher's exact test or the chi-square test as appropriate. Analysis of continuous variables was performed using Student's t-test. Data was presented as mean with standard deviation (SD), and pvalue of less than 0.05 was considered statistically significant.

\section{Results}

A total of 156 participants with respiratory infections were enrolled in this study. The mean age of the patients was $68.11 \pm 15.77$ (range 28-91) years. The mean ages for 8 B19 positive patients and 148 B19 negative ones were 67.38 and 68.18 years, respectively. According to independent t-test, there was no statistically significant differences between B19 infection and age of patients $(p=0.89)$ (Table 1). Among the 156 participants, 84 were male and 72 female. Analysis by real-time PCR assay showed that of 156 subjects, only $8(5.1 \%)$ cases were positive for parvovirus B19, including 5 males $(5 / 9 \%)$ and 3 females $(4 / 1 \%)$. According to Fisher exact test, no statistical significant differences was found between B19 and gender of patients ( $\mathrm{p}=$ 0.52) (Table 2).

The clinical manifestations of the patients are summarized in Table 3. Most prevalent complaints of patients

Table 1. Distribution of parvovirus B19 according to age of patients

\begin{tabular}{lcccc}
\hline B19 & $\mathrm{N}$ & Age (mean) & SD & $\mathrm{p}$ \\
\hline Positive & 8 & 67.38 & 11.23 & 0.89 \\
Negative & 148 & 68.18 & 16.19 & \\
\hline SD: Standard Deviation & &
\end{tabular}

Table 2. Distribution of parvovirus B19 according to gender of patients

\begin{tabular}{lccc}
\hline B19 & Male & Female & p (Fisher exact test) \\
\hline Positive & $5(5.9 \%)$ & $3(4.1 \%)$ & 0.52 \\
Negative & $79(94.1 \%)$ & $69(95.9 \%)$ & \\
\hline
\end{tabular}

Table 3. Clinical symptoms of patients with human parvovirus B19

\begin{tabular}{lcc}
\hline Symptoms & N (Percent) & p (Fisher exact test) \\
\hline Tachypnea & $8(100 \%)$ & 0.99 \\
Wheezing & $8(100 \%)$ & 0.99 \\
Pneumonia & $6(75 \%)$ & 0.99 \\
Shortness of breath & $6(75 \%)$ & 0.99 \\
Rhinorrhea/pharyngitis & $6(75 \%)$ & 0.61 \\
Fever $>38.5^{\circ} \mathrm{C}$ & $6(75 \%)$ & 0.61 \\
Cough & $5(62.5 \%)$ & 0.71 \\
Bronchitis/bronchiolitis & $5(62.5 \%)$ & 0.48 \\
Asthma & $4(50 \%)$ & 0.43 \\
Neurological disorder & $3(37.5 \%)$ & 0.25 \\
Gastrointestinal & $2(25 \%)$ & 0.61 \\
Chest retraction & $0(0 \%)$ & 0.99 \\
\hline
\end{tabular}




\begin{tabular}{ccccc} 
Table 4. Platelets and neutrophils counts in B19 positive and negative groups & \\
\hline & B19 & $\mathrm{N}$ & Mean & $\mathrm{p}$ \\
\hline Neutrophil $(\%)$ & Positive & 8 & 76.5 & 0.63 \\
& Negative & 148 & 78.3 & \\
Platelet $\left(/ \mathrm{mm}^{3}\right)$ & Positive & 8 & 222000 & 0.65 \\
& Negative & 148 & 236180 & \\
\hline
\end{tabular}

were wheezing $(100 \%)$, shortness of breath $(75 \%)$, fever (75\%), pneumonia (75\%) and rhinorrhea/pharyngitis (75\%) and in less frequency, cough $(62.5 \%)$, bronchiolitis and bronchitis $(62.5 \%)$ which were diagnosed by physicians. Also, symptoms such as gastrointestinal manifestations and neurological disorders were rarely diagnosed and no evidence of chest retraction was observed. Based on the analysis by Fisher exact test, no significant differences was observed between B19 and clinical symptoms of the patients (all $\mathrm{p}>0.05$ ).

Laboratory findings of all patients in B19 positive and negative groups, including platelets and neutrophils counts are shown in Table 4 . The numbers of platelets and neutrophils in the positive group were less in comparison with the negative group.

\section{Discussion}

Human parvovirus B19 is mainly transmitted through the respiratory route (such as saliva, sputum, or nasal mucus) and its DNA found in respiratory tract secretions at the time of viremia when infectivity is highest (5). However, infection is also transmitted vertically from mother to fetus, via blood transfusion and organ/bone marrow transplantation (11). The majority of infections with parvovirus B19 are asymptomatic or manifest non-specific, mild, cold-like symptoms. Most individuals with B19 infection do not require therapy because symptoms are mild and the illness is self-limited and resolves after 5 to 7 days (5).

The results of the present study also showed that pneumonia was a common complication in B19 positive patients $(75 \%)$. It is still unclear that lung injury mediated by direct infection or immune damage. In addition to the erythroid cells, erythrocyte $\mathrm{P}$ antigen as a cellular receptor for parvovirus B19 is found on cells of the lung tissue as well (12). Nasal congestion and rhinorrhea are frequently noted in the prodromal phase of acute B19 infection in observational studies and experimental infection $(13,14)$.

In the present study, clinical symptoms associated with B19 infection in respiratory tract were similar to previous studies. However, the association was not significant, and small sample size could probably be one if the reasons for such null-finding. It is notable that the symptoms of respiratory infections can be due to various infectious agents such as viral and bacterial infections. For this reason, we suggest that other possible co-infections be examined and in addition to a larger study population.

It should be noted that the most sensitive assay for detection of viral pathogens such as parvovirus B19 is nucleic acid based-technique especially PCR assay. Although serological assays such as ELISA can be useful for diagnosing of recent or past infection, these assays possess some limitations. In this survey, we used molecular assay instead of serologic approach due to high sensitivity and specificity, low rate of cross-reaction (particularly with rubella and rheumatoid arthritis), as well as capability to simultaneously detect acute and persistent B19 infections. This is a weak point for ELISA method which is associated with false positive results. Using probe in real-time PCR assay leads to increase the specificity and preventing false positive results (1).

Previous studies have implicated the role of the human parvovirus B19 in leucopenia, neutropenia, and thrombocytopenia (15-17). In some reports and experimental infections, it has documented that parvovirus B19 can resulted in leucopenia and neutropenia. Infection and depletion of bone marrow granulocytes may be possible reasons for this phenomenon. There are two types of B19 infection-induced thrombocytopenia. In one, due to suppression of bone marrow by the B19 infection, thrombocytopenia precedes the onset of the rash. In another one, thrombocytopenia occurs after the diagnosis of erythema infectiosum by immunological mediation (18). In the present study, we assessed the number of neutrophil and platelet and the results were compared in B19 positive and negative groups. We found that the number of platelets and neutrophils in the positive group were lower than the negative group. However, the small sample size can be potentially considered as a limitation of the study, as it reduces the study power and subsequently, the results may not generalizable to entire population of patients with respiratory infection.

\section{Conclusion}

In conclusion, this study provided the first insight into the epidemiology and clinical aspects of human B19 infection in Iranian hospitalized patients with respiratory tract infection. Our findings do not support the role of parvovirus B19 in the development of respiratory infection. However, further studies are needed to evaluate the etiological role of parvovirus B19 in respiratory infection.

\section{Acknowledgment}

This work was funded by Iran University of Medical Sciences (Grant No: 25415).

\section{Conflict of Interests}

The authors declare that they have no competing interests.

\section{References}

1. Arabzadeh SAM, Alizadeh F, Tavakoli A, Mollaei H, Bokharaei-Salim F, Karimi G, et al. Human parvovirus B19 in patients with beta thalassemia major from Tehran, Iran. Blood Res. 2017;52(1):50-54.

2. Monavari SH, Noorbakhsh S, Mollaie H, Fazlalipour M, Kiasari BA. Human Bocavirus in Iranian children with acute gastroenteritis. Med J Islam Repub Iran. 2013;27(3):127-131.

3. Shabani Z, Esghaei M, Keyvani H, Shabani F, Sarmadi F, Mollaie H, et al. Relation between parvovirus B19 infection and fetal mortality 
and spontaneous abortion. Med J Islam Repub Iran. 2015;29:1-6.

4. Zaki S. Detection of human parvovirus B19 in cancer patients using ELISA and real-time PCR. Indian J Med Microbiol. 2012;30(4): $407-$ 410.

5. Marano G, Vaglio S, Pupella S, Facco G, Calizzani G, Candura F, et al. Human Parvovirus B19 and blood product safety: a tale of twenty years of improvements. Blood Transfus. 2015;13(2):184-196.

6. Javanmard D, Ziaee M, Ghaffari H, Namaei MH, Tavakoli A, Mollaei $\mathrm{H}$, et al. Human parvovirus B19 and parvovirus 4 among Iranian patients with hemophilia. Blood Res. 2017;52(4):311-315.

7. Lysholm F, Wetterbom A, Lindau C, Darban H, Bjerkner A, Fahlander $\mathrm{K}$, et al. Characterization of the viral microbiome in patients with severe lower respiratory tract infections, using metagenomic sequencing. PloS one. 2012;7(2):e30875.

8. Giorgio E, De Oronzo MA, Iozza I, Di Natale A, Cianci S, Garofalo G, et al. Parvovirus B19 during pregnancy: a review. J Perinat Med. 2010;4(4):63-66.

9. Rogo L, Mokhtari-Azad T, Kabir M, Rezaei F. Human parvovirus B19: a review. Acta Virol. 2014;58(3):199-213.

10. Romero-Espinoza JA, Moreno-Valencia Y, Coronel-Tellez RH, Castillejos-Lopez M, Hernandez A, Dominguez A, et al. Virome and bacteriome characterization of children with pneumonia and asthma in Mexico City during winter seasons 2014 and 2015. PloS one. 2018;13(2):e0192878.

11. Lamont RF, Sobel J, Vaisbuch E, Kusanovic JP, Mazaki-Tovi S, Kim SK, et al. Parvovirus B19 infection in human pregnancy. BJOG. 2011;118(2):175-186.

12. Costa C, Terlizzi ME, Solidoro P, Libertucci D, Bergallo M, Cavallo R. Detection of parvovirus B19 in the lower respiratory tract. J Clin Virol. 2009;46(2):150-153.

13. Kazama I, Sasagawa N, Nakajima T. Complete remission of human parvovirus b19 associated symptoms by loxoprofen in patients with atopic predispositions. Case Rep Med. 2012;2012:1-4.

14. Shih H-I, Wang H-C, Su I-J, Hsu H-C, Wang J-R, Sun HFS, et al. Viral Respiratory Tract Infections in Adult Patients Attending Outpatient and Emergency Departments, Taiwan, 2012-2013: A PCR/Electrospray Ionization Mass Spectrometry Study. Medicine. 2015;94(38):1-9.

15. Yaguchi D, Marui N, Matsuo M. Three adult cases of HPV-b19 infection with concomitant leukopenia and low platelet counts. Clin Med Insights Case Rep. 2015;8:CCRep. S18085.

16. van Asseldonk DP, Kanis BM, de Boer NK, van Bodegraven AA. Leukopenia due to parvovirus B19 in a Crohn's disease patient using azathioprine. Digestion. 2009;79(4):211-214.

17. Frotscher B, Salignac S, Morlon L, Bonmati C, Jeulin H, Venard V, et al., editors. Neutropenia and/or thrombocytopenia due to acute parvovirus B19 infection. Ann Biol Clin; 2009;67(3):343-348.

18. Nagaharu $K$, Sugimoto $Y$, Hoshi $Y$, Yamaguchi $T$, Ito $R$, Matsubayashi K, et al. Persistent symptomatic parvovirus B19 infection with severe thrombocytopenia transmitted by red blood cell transfusion containing low parvovirus B19 DNA levels. Transfusion. 2017;57(6):1414-1418 